\title{
Modelos Acoplados do IPCC (CMIP3-CMIP5) e o Gradiente Meridional de Anomalias de Temperatura da Superfície do Mar (TSM) no Oceano Atlântico Tropical
}

\author{
Érika A. Assis ${ }^{1}$ (D), José Maria B. Alves ${ }^{1}$ (D), Emerson M. Silva ${ }^{1}$ (D), \\ Francisco das C. Vasconcelos Júnior ${ }^{2}$ (D), Augusto C.B. Barbosa ${ }^{1}$ (D), \\ Antônio C. Santana dos Santos $^{1}$ (D), Sérgio S. Sombra ${ }^{1}$ \\ ${ }^{1}$ Departamento de Física, Universidade Estadual do Ceará, Fortaleza, CE, Brasil. \\ ${ }^{2}$ Fundação Cearense de Meteorologia e Recursos Hídricos, Fortaleza, CE, Brasil.
}

Recebido em: 9 de Setembro de 2018 - Aceito em: 3 de Fevereiro de 2019

\begin{abstract}
Resumo
Este estudo investigou a sensibilidade de modelos acoplados oceano-atmosfera do CMIP3 e do CMIP5 para o período de 1979-1999 em simular a magnitude do gradiente meridional de anomalias de Temperaturas da Superfície do Mar (TSM) no Atlântico Tropical. Nos resultados são apresentadas análises comparativas para este gradiente meridional de anomalias de TSM de dois períodos sazonais (verão e outono austrais). Os resultados mostraram a evolução dos modelos em simular as variações das anomalias de TSM desse gradiente do CMIP3 para o CMIP5. Para o CMIP3 os modelos não tiveram um bom desempenho em ambas as estações. Para o CMIP5 o verão apresentou o maior número de modelos com índice de desempenho de Willmott (igual ou acima de mediano ID $\geq 0,6$ ).
\end{abstract}

Palavras-chave: TSM, anomalias de TSM, Atlântico Tropical, modelos acoplados.

\section{IPCC Coupled Models (CMIP3-CMIP5) and the Southern Ocean Surface Temperature Anomaly Gradient (SST) in the Tropical Atlantic Ocean}

\begin{abstract}
This study investigated the sensitivity models coupled ocean-atmosphere of CMIP3 and CMIP5 for the period of 19791999 to simulate the magnitude of the meridional gradient of sea surface temperature anomalies (SST) in Atlantic Tropical. In the results are presented to comparative analyses for this meridional gradient of SST anomalies of two seasonal seasons (summer and autumn autrais). The results showed the evolution of the models in simulating the variations of SST anomalies of this gradient from CMIP3 to CMIP5. For CMIP3 the models did not good perform in both seasons. For the CMIP5 the summer presented the highest number of models with Willmott performance index (equal to or above the median ID $\geq 0.6$ ).
\end{abstract}

Keywords: SST, SST anomalies, climate change, coupled models.

\section{Introdução}

A média das condições do tempo ao longo de um período de algumas décadas denomina-se clima. O clima de certa região é determinado por um conjunto de vários fatores, tais como sua distância aos oceanos, a altitude do local e a distância deste ao equador. O ar e a água são variáveis determinantes para o clima da Terra. Com dinâmica própria, ambos podem interagir entre si e com o meio (Cavalcanti et al., 2009). Nesse contexto, vale ressaltar a importância da variabilidade da temperatura superficial das águas (TSM) do Oceano Atlântico, que afeta o clima dos continentes próximos e consequentemente sua população.

Autor de correspondência: José M.B. Alves, braboalves@gmail.com. 
O clima não mantém exatamente o mesmo comportamento para uma determinada área, pois existem as anomalias, que diferem do comportamento "padrão" climático de uma região. Acerca da variabilidade térmica dos oceanos alguns modos, também chamados de gradientes de anomalias de TSM, são responsáveis por anomalias climáticas, em áreas do Caribe, áfrica e América do Sul (Hastenrath, 1991, Nobre e Shukla, 1996, Servain et al., 2002, 2009; Alves et al., 2009 e 2012, 2014). Entre esses modos de variabilidade destacam-se o gradiente meridional de anomalias de TSM, também chamado de modo de dipolo meridional de anomalias de TSM, o modo zonal ou equatorial de anomalias de TSM e o modo subtropical de anomalias de TSM, sendo que este também pode ser denominado de SAD (South Atlantic Dipole). Neste estudo apenas modo denominado de gradiente meridional de anomalias de TSM do Atlântico Tropical será analisado. Ao longo do texto este modo pode ser chamado apenas de modo gradiente meridional ou dipolo meridional.

Há também outros dipolos, como o dipolo de anomalias de TSM do Oceano índico, que também têm sido foco de estudos. Saji et al. 1999 demonstra que os eventos deste dipolo estão associados a mudanças de larga escala da atmosfera sobre o Oceano índico equatorial e têm sido relacionados a anomalias de precipitação em vários locais vizinhos a esse oceano, incluindo a ásia Oriental (Guan e Yamagata, 2003) e a Austrália (Ashok e Yamagata. 2003). Porém, esse dipolo não é objeto desse estudo.

O modo de dipolo ou gradiente meridional de anomalias de TSM é uma dessas mudanças térmicas anômalas, sendo este, na TSM no Oceano Atlântico Tropical. Este oceano possui uma complexa variabilidade física intrassazonal, anual e interanual com alguns modos de variabilidade térmicos principais que definem a chamada Variabilidade do Atlântico Tropical (TAV - Tropical Atlantic Variability, Tanimoto e Xie, 2002). Esse fenômeno causa alterações na célula de Hadley (célula meridional de convecção) e pode inibir ou aumentar a formação de nuvens, em particular, no Nordeste Brasileiro (NEB) e em alguns países da áfrica, alterando a precipitação nessas regiões.

O modo meridional caracteriza-se, em geral, por dipolo de anomalias de TSMs entre as áreas norte e sul do Atlântico Tropical. O modo zonal ou gradiente equatorial de anomalias de TSM manifesta-se como anomalias de TSM na faixa equatorial do Oceano Atlântico, entre os seus setores leste e oeste. Ambos os modos associam-se à circulação atmosférica sobre o equador e vizinhanças, e também tem sido assunto de vários estudos (Enfield et al. 1999; Chiang e Vimont, 2004).

O SAD tem suas variações de anomalias de TSM concentradas em duas áreas a sudeste e noroeste do Atlântico Subtropical. Estudos têm mostrado a relação da variabilidade desse dipolo de TSM e o clima das regiões
Sudeste e Sul da América do Sul, principalmente (Tascheto e Wainer, 2008 e Nnamchi et al. 2011).

O Painel Intergovernamental de Mudanças Climáticas (Intergornmental Pannel on Climate Change -IPCC) é o principal órgão científico internacional para as avaliações de estudo sobre as alterações climáticas. Nesses últimos oito anos o IPCC publicou dois relatórios, o AR4 e o AR5 (Assessments Reports Fourth and Fifth Phases), e utilizou dados modelados e projeções futuras do CMIP3, e do CMIP5 (do inglês o Projeto de Intercomparação de Modelos Acoplados, em suas terceira e quinta fases, respectivamente) do Programa de Pesquisa Climática Mundial, o WCRP (World Climate Research Programme). Para isso, diversos centros de modelagem climática submeteram seus resultados (de simulações e em formatos padrões) para o Programa de Diagnóstico e Intercomparação de Modelos Climáticos (PCMDI - Program for Climate Model Diagnosis and Intercomparison). No PCMDI são analisados quais MCG (Modelos Climáticos Globais acoplados com o oceano ou não) conseguem representar de forma satisfatória o clima global atual, de tal maneira que tais modelos possam simular o futuro sem produzir muitas incertezas relativas aos erros produzidos que esses modelos no clima presente.

No intuito de facilitar a modelagem climática e a avaliação dos impactos, o IPCC criou cenários de projeções baseados nas emissões e nas concentrações de $\mathrm{CO}_{2}$ (MOSS et al,. 2010). Para o CMIP5 foram feitos quatro cenários denominados de RCP's (do inglês, Representative Concentration Pathways), onde estes fornecem uma gama de simulações para o futuro e assim caracterizando as próximas décadas em função de forçantes radiativas. Estas projeções podem ser usadas como base para explorar os impactos das alterações climáticas e as questões políticas de considerável interesse e relevância para a sociedade (Taylor et al. 2010).Os quatro cenários utilizados no quinto relatório do IPCC diferem de acordo com as características radiativas do sistema climático.

Dessa forma, e em face às mudanças climáticas vide relatórios do Painel Intergovernamental de Mudanças Climáticas (IPCC, 2007 e 2013) - estudos que aprofundem o conhecimento das variabilidades desses modos gradientes de anomalias de TSM no Atlântico, citados anteriormente, em particular como os modelos os representam e as suas projeções para o futuro poderão ser de extrema importância para subsídios que se referem aos impactos climáticos nessas regiões da América do Sul, áfrica e Caribe, podendo vir a nortear planejamentos nas áreas econômica, social e política.

Nesse contexto, o objetivo deste estudo foi investigar a magnitude do gradiente meridional de anomalias de TSM no Oceano Atlântico Tropical e como os modelos acoplados do CMIP3 e do CMIP5 simulam as características desse gradiente, com ênfase no verão e outono austral (1979-1999). 


\section{Materiais e Métodos}

Os dados de TSM observados usados nesse estudo são do ERSST (Extended Reconstructed Sea Surface Temperature), para o período de 1979 - 1999. Esse dado é resultado do método da "Reconstrução Estendida", desenvolvido por Smith e Reynolds (2002), para estimar campos de TSM. Estes dados foram reconstruídos desde 1854 até o presente, sendo que a partir de 1880 esses dados se tornaram mais consistentes. A resolução espacial desses dados de TSM reconstruída é de $2^{\circ} \times 2^{\circ}$ de latitude e longitude, com integridade espacial aumentada usando métodos estatísticos, e a base temporal da reconstrução estendida é mensal. A versão mais recente do ERSST, versão quatro, é baseada em parâmetros otimizados, utilizando os mais recentes conjuntos de dados e melhorias dos métodos de análise (Huang et al. 2015; Smith et al. 2008).

Os dados de TSM simulados e projetados referem-se aos modelos oceano-atmosfera acoplados do IPCC, do AR4 e do AR5, em períodos simulados entre 1979 - 1999. Detalhes desses modelos quanto à resolução espacial, e centros meteorológicos responsáveis por suas rodadas, forçantes podem ser vistas em Taylor et al. 2012 e Meehl et al. 2007. Quanto a detalhes sobre o volume de gases de efeito estufa nas rodadas de simulação e projeção dos modelos do AR4 e AR5 podem ser encontrados também em Taylor et al. 2012 e Meehl et al. Em geral, as rodadas antes do ano 2000, tem como base a concentração de gases de efeito estufa da ordem de 320 ppm de $\mathrm{CO}_{2}$.

Para as comparações entre os dados observados e simulados foram calculadas algumas variáveis para 0 verão e outono austrais das suas áreas norte e sul representativas dos gradiente meridional de anomalias de TSM (norte $-60^{\circ} \mathrm{W}-30^{\circ} \mathrm{W}$ e $5^{\circ} \mathrm{N}-20^{\circ} \mathrm{N}$ e sul $-30^{\circ} \mathrm{W}-10^{\circ} \mathrm{W}$ e $5^{\circ} \mathrm{S}-20^{\circ} \mathrm{S}$ ), As variáveis utilizadas foram: as médias das TSM (1979-1999) nessas áreas norte e sul, anomalias, que é a média em cada área de cada ano menos a sua média para cada estação, o cálculo do índice dos gradientes meridional, que é dado pela diferença das anomalias na área norte menos as respectivas anomalias da área sul.

Ressalta-se que as estatísticas descritas a seguir foram feitos para escalas mensais para os meses das estações do ano - verão: dezembro a fevereiro, outono: março a maio.

As categorias dos gradientes de anomalias de TSM foram divididas em dipolo positivo (DipPos), dipolo negativo (DipNeg), dipolo neutro (DipNeut). Quando a diferença na área norte menos a área sul do referido gradiente de anomalias de TSM mencionado, acima for menor ou igual a $-0,2{ }^{\circ} \mathrm{C}$ têm-se um dipolo negativo, para o dipolo neutro a diferença dever ser maior que $-0,2{ }^{\circ} \mathrm{C}$ e menor ou igual a $0,2{ }^{\circ} \mathrm{C}$ e para dipolo positivo a diferença deve ser maior que $0,2{ }^{\circ} \mathrm{C}$. Essas categorias foram identificadas anualmente para cada estação de verão e outono.

A seguir são colocadas algumas métricas que foram usadas nos cálculos citados acima e outras que servirão para identificar que modelos acoplados são mais sensíveis em reproduzir as variações dos índices de dipolo nos gradientes de anomalias de TSM meridional no período 19791999.

Depois de obtidos os resultados dessas métricas são avaliados quantos dipolos positivos, negativos e neutros coincidiram com o seu respectivo no dado observado. Dessa forma se infere se um determinado modelo acoplado apresentar cinco dipolos positivos coincidentes isso significa que este modelo simulou esse dipolo no mesmo período ocorrido na observação.

A seguir serão abordadas as métricas estatísticas utilizadas para a obtenção dos resultados deste trabalho. A Média Aritmética, dada na Eq. (1), corresponde aos somatórios dos $\mathrm{n}$ elementos divididos pelo tamanho da amostra, em $n$ tempos.

$$
X_{m}=\frac{1}{n} \sum_{i=1}^{i=n} x_{i}
$$

onde $X_{m}$ é a média, n é o número da população ou amostra usada e $x_{i}$ é o valor da variável correspondente analisada.

A anomalia calculada é resultado da diferença entre o valor de um elemento da série menos a média aritmética dessa série, no caso uma amostra. Sua formulação matemática é dada na Eq. (2).

$$
A_{m}=x_{i}-X_{m}
$$

A variância $\left(S^{2}\right)$ e o desvio padrão $(\sigma)$ são métricas utilizadas para medir o grau de dispersão das variáveis de uma determinada amostra em torno de um valor central. A medida de dispersão mais utilizada é o desvio padrão, que é definido pela raiz quadrada da variância. Onde a variância é dada pela Eq. (3).

$$
S^{2}=\frac{1}{n} \sum_{i=1}^{i=n}\left(x_{i}-X_{m}\right)^{2}
$$

O desvio padrão $(\sigma)$ é dado pela Eq. (4).

$$
\sigma=\sqrt{S^{2}}
$$

O coeficiente de Correlação de Pearson ( $r$ ) infere uma associação bivariada do grau de relacionamento entre duas variáveis, no caso X e Y. Isto é, trata-se de uma medida de associação linear entre elas. Sua formulação matemática é dada pela Eq. (5).

$$
r_{x y}=r=\frac{\operatorname{Cov}(x, y)}{\sigma_{x} \sigma_{y}}
$$

O coeficiente tem um caráter adimensional, ou seja, ele é desprovido de unidade física que o defina. Os valores de $r$ 
estão limitados entre uma escala de -1 até +1 , onde o valor indica a direção da relação linear, geralmente, a força relativa de uma relação linear, ou seja, a adequação com que a relação linear descreve os dados. Valores de $r$ geralmente na vizinhança tanto de -1 quanto de +1 indicam uma relação relativamente forte, e valores de $r$ na vizinhança de 0 (zero) indicam uma relação fraca ou quase inexistente (Witte e Witte, 2005). Ver Tabela 1.

O Erro Quadrático médio $\left(E q_{m}\right)$, dado pela Eq. (6). Este indica o grau de dispersão das variáveis de uma determinada amostra em torno de um valor central, porém não leva em consideração a diferença ao quadrado entre os mesmos.

$$
E q_{m}=\frac{1}{n-1} \sum_{i=1}^{i=n} \sqrt{\left(x_{i}-X_{m}\right)^{2}}
$$

Outra métrica utilizada como base padrão definitiva da escolha dos melhores modelos é o índice de desempenho (ID). Este índice avalia o desempenho dos modelos em estudo, pois reúne os índices de precisão, dado pelo coeficiente de correlação $(r)$ que indica o grau de dispersão dos dados obtidos em relação à média, ou seja, o erro aleatório e o de concordância (índice de Willmott) $d$. O índice $I D$ é calculado conforme a Eq. (7).

$$
I D=r . d
$$

O valor do índice de Willmott é dado pela Eq. (8).

$$
d=1-\frac{\sum_{i=1}^{i=n}\left|O_{i}-P_{i}\right|^{2}}{\sum_{i=1}^{i=n}\left(\left|P_{i}-O_{m}\right|+\left|O_{i-} O_{m}\right|\right)^{2}}
$$

A exatidão está relacionada ao afastamento dos valores estimados $\left(P_{i}\right)$ em relação aos observados $\left(O_{i}\right)$, é dada estatisticamente por este índice de concordância d proposto por Willmott et al. 1985. Seus valores variam de zero, para nenhuma concordância, a um, para a concordância perfeita. O índice $O_{m}$ na Eq. (8) significa média dos valores da variável observada.

Tabela 1 - Análise qualitativa do grau de correlação entre duas variáveis. Fonte: Witte e Witte, 2005.

\begin{tabular}{lc}
\hline$R$ & Magnitude da correlação \\
\hline 0,0 & Nula \\
$0,0 \leq r \leq 0,3$ & Fraca \\
$0,3 \leq r \leq 0,6$ & Moderada \\
$0,6 \leq r \leq 0,9$ & Forte \\
$0,9 \leq r \leq 1,0$ & Muito Forte \\
1,0 & Perfeita \\
\hline
\end{tabular}

$\mathrm{O}$ índice de desempenho pode ser classificado segundo a Tabela 2 (Willmott et al. 1985).

\section{Resultados e Discussões}

Os resultados deste trabalho estão descritos a seguir de acordo com o período sazonal (DJF - Verão, MAM Outono). Primeiramente serão apresentados os resultados dos modelos climáticos que compõem o CMIP3. A mesma avaliação é feita para os modelos que fazem parte do CMIP5 para o período de 1979-1999.

As análises abaixo mostram o comportamento dos modelos do CMIP3 para o período 1979-1999 onde identifica-se a presença do gradiente meridional de anomalias de TSM em suas três fases (positiva negativa e neutra) interanualmente comparado as observações para o verão e outono austrais.

A Fig. 1 mostra a quantidade de eventos do gradiente meridional para o trimestre de DJF (dezembro, janeiro e fevereiro). As primeiras três barras apresentam o dado observado. Este apresenta oito dipolos positivos e negativos e cinco dipolos neutros. Os modelos apresentaram uma média, de seis dipolos positivos e seis negativos, e nove dipolos neutros. Neste caso o gradiente meridional em suas fases positiva e negativa foi subestimado, mas a fase neutra foi superestimada. O modelo HADCM3 foi o único modelo que acertou a quantidade de eventos classificados como neutro, e o restante deles superestimou o número de eventos dessa fase.

Os modelos CSIRO_MK3 0, ECHAM5, HADCM3 e HADGEM1 simularam corretamente a quantidade de eventos negativos. Os que acertaram o evento do gradiente meridional positivo foram CGCM2_3_2A e HADCM3. O HADCM3 simulou igual ao observado a quantidade desse dipolo em suas três fases.

A Tabela 3 apresenta as métricas estatísticas para a estação verão. Todas elas foram obtidas em relação ao dado observado ERSST. Observa-se que nenhum modelo apresentou ID maior que 0,6 , ou seja, nenhum modelo apresentou desempenho igual ou superior ao mediano em relação a este parâmetro, apesar de alguns modelos como CNRM_CM3 e o CSIRO_MK3_0 passarem no teste tStudent para a correlação.

Tabela 2 - Critério de interpretação do desempenho do ID.

\begin{tabular}{lc}
\hline ID & Desempenho \\
\hline$>0,85$ & ótimo \\
0,76 a 0,85 & Muito Bom \\
0,66 a 0,75 & Bom \\
0,61 a 0,65 & Mediano \\
0,51 a 0,60 & Sofrível \\
0,41 a 0,50 & Mau \\
$\leq 0,40$ & Péssimo \\
\hline
\end{tabular}




\section{Gradiente Meridional - Verão do AR4}

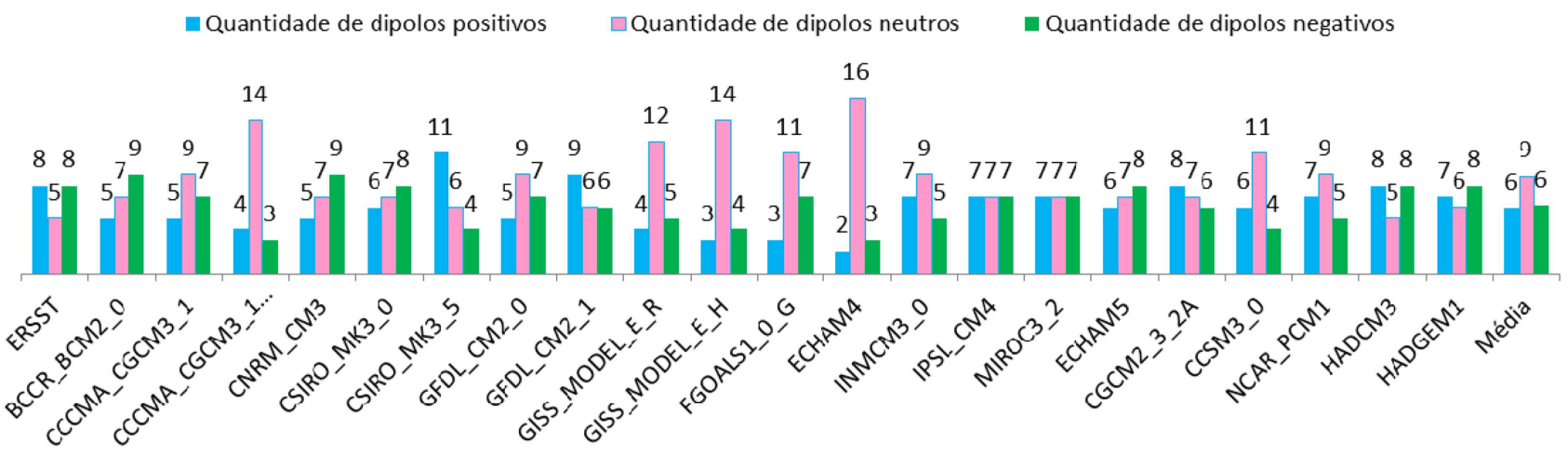

Figura 1. - Eventos do gradiente meridional de anomalias de TSM para a estação de verão dos dados do ERSST e dos modelos CMIP3 (1979-1999). As barras verdes, azuis e rosas apresentam a quantidade de dipolos negativos, positivos e neutros, respectivamente.

A Fig. 2 mostra a quantidade de eventos do gradiente meridional para cada modelo do quarto relatório do IPCC, que compõem o CMIP3 para a estação de outono comparado as observações. Para as observações notam-se quatro dipolos positivos, três dipolos negativos e quatorze dipolos neutros. Os modelos apresentaram uma média de dois dipolos positivos e negativos, e dezessete dipolos neutros, ou seja, os modelos como um todo subestimaram a quantidade de dipolos positivos e negativos e superestimaram o dipolo neutro.

Os modelos INMCM3 0, NCAR_PCM1 e HADGEM1 simularam corretamente a quantidade de eventos negativos. Apenas o modelo INMCM3 0 acertou na quantidade de dipolos positivos (quatro) e os modelos CSIRO_MK3_5, INMCM3_0 e HADCM3 foram exatos em simula a frequência do dipolo em sua fase neutra.

A Tabela 4 apresenta as métricas estatísticas para a estação outono para os modelos do CMIP3. Todas elas foram obtidas em relação ao dado observado ERSST.
Pode-se observar que dois modelos apenas apresentaram ID classificado como de bom (superior a 0,60), o. CCCMA_CGCM3_1_T63 e o GFDL_CM2_1.

A Fig. $3(a, b)$ mostram o número de eventos simulados e observados para a estação do verão comparado as observações para os modelos do CMIP5 (1979-1999). Os resultados da observação indicam a incidência de oito dipolos positivos, oito dipolos negativos e cinco dipolos neutros. Os modelos apresentaram média de seis dipolos positivos e cinco negativos, e onze dipolos neutros. A fase neutra foi a menos consistente com a observação. Os modelos FGOALS-g2, IPSL-CM5A-LR, MIROC-ESMCHEM foram os únicos que subestimaram a quantidade de eventos classificados como neutro, sendo que apenas um modelo refletiu exatamente essa fase, o INMCM4_0.

Os modelos FGOALS-g2, HadCM3, IPSL-CM5ALR, IPSL-CM5A-MR simularam corretamente a quantidade de eventos negativos. Para o gradiente meridional positivo apenas quatro modelos conseguiram simular

\section{Gradiente Meridional - Outono do AR4}

— Quantidade de dipolos positivos $\quad \square$ Quantidade de dipolos neutros Q Quantidade de dipolos negativos

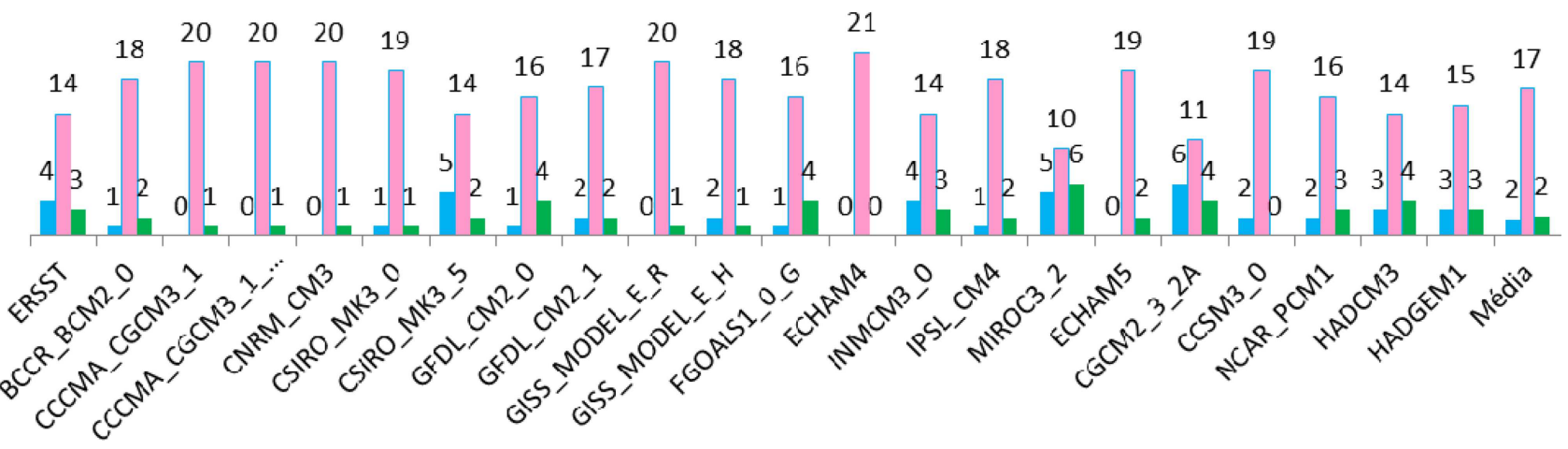

Figura 2. - Eventos do gradiente meridional de anomalias de TSM para a estação de outono dos dados do ERSST e dos modelos do CMIP3 (1979-1999). As barras verdes, azuis e rosas apresentam a quantidade de dipolos negativos, positivos e neutros, respectivamente. 
Tabela 3 - Estatísticas para a estação de verão, modo meridional, entre os modelos e os dados do ERSST (1979-1999). As linhas em negrito indicam quando o coeficiente de correlação foi significativo segundo teste $\mathrm{t}$ Student a $95 \%$.

\begin{tabular}{lcccc}
\hline Modelos do AR4 - Verão & $\begin{array}{c}\text { Erro } \\
\text { Médio } \\
\left({ }^{\circ} \mathrm{C}\right)\end{array}$ & $\begin{array}{c}\text { Erro } \\
\text { Quadrático } \\
\left({ }^{\circ} \mathrm{C}\right)\end{array}$ & ID & Correlação \\
\hline BCCR_BCM2_0 & $\mathbf{- 0 , 1 2}$ & $\mathbf{0 , 1 3}$ & $\mathbf{- 0 , 4 7}$ & $\mathbf{- 0 , 5 7}$ \\
CCCMA_CGCM3_1 & $-0,03$ & 0,09 & $-0,22$ & $-0,27$ \\
CCCMA_CGCM3_1_T63 & $\mathbf{0 , 0 4}$ & $\mathbf{0 , 0 7}$ & $\mathbf{0 , 4 5}$ & $\mathbf{0 , 5 4}$ \\
CNRM_CM3 & $\mathbf{- 0 , 0 9}$ & $\mathbf{0 , 1 3}$ & $\mathbf{- 0 , 4 1}$ & $\mathbf{- 0 , 5 1}$ \\
CSIRO_MK3_0 & $\mathbf{- 0 , 0 7}$ & $\mathbf{0 , 1 5}$ & $\mathbf{- 0 , 4 7}$ & $\mathbf{- 0 , 6 0}$ \\
CSIRO_MK3_5 & 0,11 & 0,11 & 0,24 & 0,30 \\
GFDL_CM2_0 & $-0,12$ & 0,23 & $-0,13$ & $-0,22$ \\
GFDL_CM2_1 & $\mathbf{0 , 1 2}$ & $\mathbf{0 , 1 5}$ & $\mathbf{0 , 2 7}$ & $\mathbf{0 , 3 9}$ \\
GISS_MODEL_E_R & $-0,02$ & 0,07 & $-0,06$ & $-0,07$ \\
GISS_MODEL_E_H & $-0,02$ & 0,08 & $-0,02$ & $-0,02$ \\
FGOALS1_0_G & $-0,10$ & 0,09 & $-0,27$ & $-0,34$ \\
CHAM4 & 0,01 & 0,04 & $-0,04$ & $-0,04$ \\
INMCM3_0 & 0,06 & 0,15 & 0,13 & 0,18 \\
IPSL_CM4 & $\mathbf{0 , 0 1}$ & $\mathbf{0 , 1 1}$ & $\mathbf{0 , 5 1}$ & $\mathbf{0 , 6 7}$ \\
MIROC3_2 & 0,00 & 0,28 & $-0,11$ & $-0,16$ \\
ECHAM5 & $-0,05$ & 0,10 & $-0,09$ & $-0,11$ \\
CGCM2_3_2 & $\mathbf{0 , 0 3}$ & $\mathbf{0 , 1 5}$ & $\mathbf{0 , 3 4}$ & $\mathbf{0 , 5 0}$ \\
CCSM3_0 & 0,03 & 0,08 & 0,21 & 0,27 \\
NCAR_PCM1 & 0,02 & 0,12 & 0,26 & 0,33 \\
HADCM3 & $\mathbf{0 , 0 4}$ & $\mathbf{0 , 3 3}$ & $\mathbf{- 0 , 2 3}$ & $\mathbf{- 0 , 3 8}$ \\
HADGEM1 & $-0,08$ & 0,51 & $-0,06$ & $-0,12$ \\
Média & $-0,02$ & 0,15 & $-0,01$ & $-0,01$ \\
\hline
\end{tabular}

corretamente, são eles: FIO-ESM, HadGEM2-AO, MIROC-ESM, IROC-ESM-CHEM.

A Tabela 5 apresenta as métricas - erro médio, erro quadrático médio, ID e correlação - para a estação verão entre os modelos do CMIP5 e os dados ERRST. Todas elas foram obtidas em relação ao dado ERSST. Observa-se que oito modelos apresentaram ID maior que 0,6 (BCC_CSM1_1m, BNU_ESM, CMCC_CESM, CMCC CM5, GISS-E2-H, HadCM3, IPSL-CM5A-LR, MIROC-ESM).

Dentre modelos citados acima três apresentaram ID ótimo e muito bom. O modelo CMCC_CESM com desempenho igual a 0,84 foi classificado como ótimo e os modelos BCC_CSM1_1m e CMCC_CM5 apresentaram ID de 0,84 e 0,77 , considerado ótimo.

Fig. $4(a, b)$ mostram a quantidade de eventos do gradiente meridional de anomalias de TSM para cada modelo do quinto relatório do IPCC, que compõem o CMIP5 para a estação de outono austral. Nas observações (ERSST) foram identificados quatro dipolos positivos, três dipolos negativos e quatorze dipolos neutros. Os modelos
Tabela 4 - Estatísticas para a estação de outono, gradiente meridional de anomalias de TSM, entre os modelos do CMIP3 e os dados do ERSST (1979-1999). Os modelos com maiores IDs e coeficiente de correlação estão marcados em amarelo. As linhas em amarelo indicam quando o coeficiente de correlação foi significativo segundo teste t Student a 95\%.

\begin{tabular}{lcccc}
\hline Modelos do AR4 - Outono & $\begin{array}{c}\text { Erro } \\
\text { Médio } \\
\left({ }^{\circ} \mathrm{C}\right)\end{array}$ & $\begin{array}{c}\text { Erro } \\
\text { Quadrático } \\
\left({ }^{\circ} \mathrm{C}\right)\end{array}$ & ID & Correlação \\
\hline BCCR_BCM2_0 & $-0,02$ & 0,32 & 0,27 & 0,31 \\
CCCMA_CGCM3_1 & $\mathbf{- 0 , 0 2}$ & $\mathbf{0 , 3 6}$ & $\mathbf{- 0 , 7 9}$ & $\mathbf{- 0 , 8 3}$ \\
CCCMA_CGCM3_1_T63 & $\mathbf{0 , 0 1}$ & $\mathbf{0 , 2 5}$ & $\mathbf{0 , 7 5}$ & $\mathbf{0 , 8 0}$ \\
CNRM_CM3 & $-0,04$ & 0,34 & 0,12 & 0,13 \\
CSIRO_MK3_0 & 0,01 & 0,47 & $-0,29$ & $-0,31$ \\
CSIRO_MK3_5 & $\mathbf{0 , 0 8}$ & $\mathbf{0 , 6 3}$ & $\mathbf{0 , 4 1}$ & $\mathbf{0 , 4 9}$ \\
GFDL_CM2_0 & $\mathbf{- 0 , 0 9}$ & $\mathbf{0 , 4 7}$ & $\mathbf{- 0 , 5 5}$ & $\mathbf{- 0 , 6 9}$ \\
GFDL_CM2_1 & $\mathbf{0 , 0 6}$ & $\mathbf{0 , 3 8}$ & $\mathbf{0 , 6 6}$ & $\mathbf{0 , 7 8}$ \\
GISS_MODEL_E_R & $\mathbf{- 0 , 0 4}$ & $\mathbf{0 , 3 1}$ & $\mathbf{- 0 , 6 6}$ & $\mathbf{- 0 , 7 2}$ \\
GISS_MODEL_E_H & $\mathbf{0 , 0 3}$ & $\mathbf{0 , 3 1}$ & $\mathbf{- 0 , 4 4}$ & $\mathbf{- 0 , 4 9}$ \\
FGOALS1_0_G & $\mathbf{- 0 , 1 1}$ & $\mathbf{0 , 3 8}$ & $\mathbf{- 0 , 5 0}$ & $\mathbf{- 0 , 6 1}$ \\
ECHAM4 & $\mathbf{0 , 0 1}$ & $\mathbf{0 , 3 2}$ & $\mathbf{0 , 5 7}$ & $\mathbf{0 , 6 0}$ \\
INMCM3_0 & 0,02 & 0,60 & $-0,02$ & $-0,02$ \\
IPSL_CM4 & $\mathbf{0 , 0 3}$ & $\mathbf{0 , 2 8}$ & $\mathbf{0 , 3 3}$ & $\mathbf{0 , 4 0}$ \\
MIROC3_2 & $\mathbf{- 0 , 0 4}$ & $\mathbf{0 , 5 7}$ & $\mathbf{- 0 , 3 0}$ & $\mathbf{- 0 , 3 9}$ \\
ECHAM5 & $-0,03$ & 0,35 & 0,14 & 0,16 \\
CGCM2_3_2 & $\mathbf{0 , 0 6}$ & $\mathbf{0 , 3 4}$ & $\mathbf{0 , 5 8}$ & $\mathbf{0 , 7 8}$ \\
CCSM3_0 & 0,04 & 0,32 & $-0,31$ & $-0,34$ \\
NCAR_PCM1 & $\mathbf{- 0 , 0 1}$ & $\mathbf{0 , 3 9}$ & $\mathbf{0 , 5 3}$ & $\mathbf{0 , 6 0}$ \\
HADCM3 & $\mathbf{- 0 , 0 5}$ & $\mathbf{0 , 7 1}$ & $\mathbf{- 0 , 6 2}$ & $\mathbf{- 0 , 7 7}$ \\
HADGEM1 & $-0,11$ & 0,59 & $-0,20$ & $-0,34$ \\
Média & $-0,01$ & 0,41 & $-0,02$ & $-0,02$ \\
\hline & & & & \\
\hline
\end{tabular}

apresentaram uma quantidade de dois dipolos positivos e dois negativos, e dezessete dipolos neutros, ou seja, os modelos como um todo subestimaram a quantidade de dipolos positivos e negativos e superestimaram o dipolo neutro.

Os modelos EC EARTH, HadGEM2-AO, IPSLCM5A-MR simularam corretamente a quantidade de eventos negativos. Quatro modelos acertaram na quantidade de dipolos positivos: BCC_CSM1_1, CSIRO_MK3_6, IPSL-CM5A-MR e os modelos BNU_ESM e IPSL-CM5A-MR foram exatos em simular a frequência do dipolo em sua fase neutra.

A Tabela 6 apresenta as estatísticas de desempenho dos modelos do CMIP5 em relação ao dado de ERSST (1979-1999) para o outono. Os modelos que estão em destaque - com realce amarelo - apresentaram valores de ID (índice de desempenho) acima de 0,61 são considerados medianos e os modelos em negrito apresentam correlações significativas iguais a $95 \%$, de acordo com o teste-t de Student. 


\section{a) Gradiente Meridional - Verão do AR5}

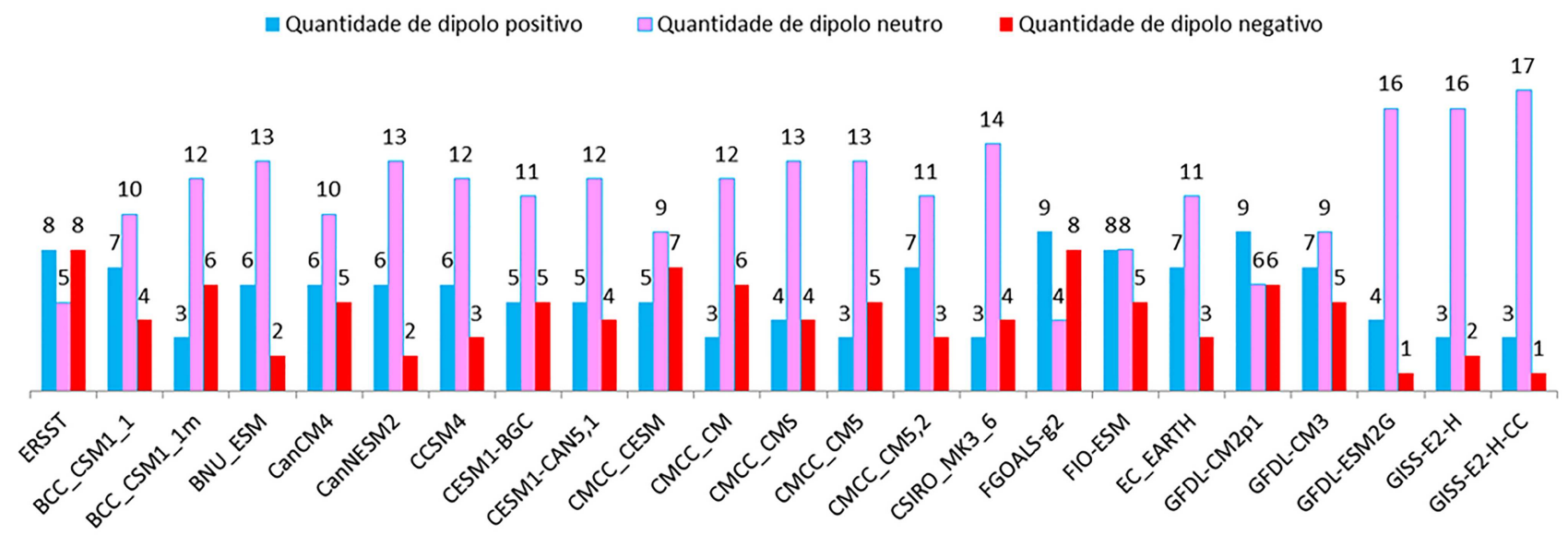

b) Gradiente Meridional- Verão do AR5

घ Quantidade de dipolo positivo $\quad \square$ Quantidade de dipolo neutro $\quad$ Quantidade de dipolo negativo

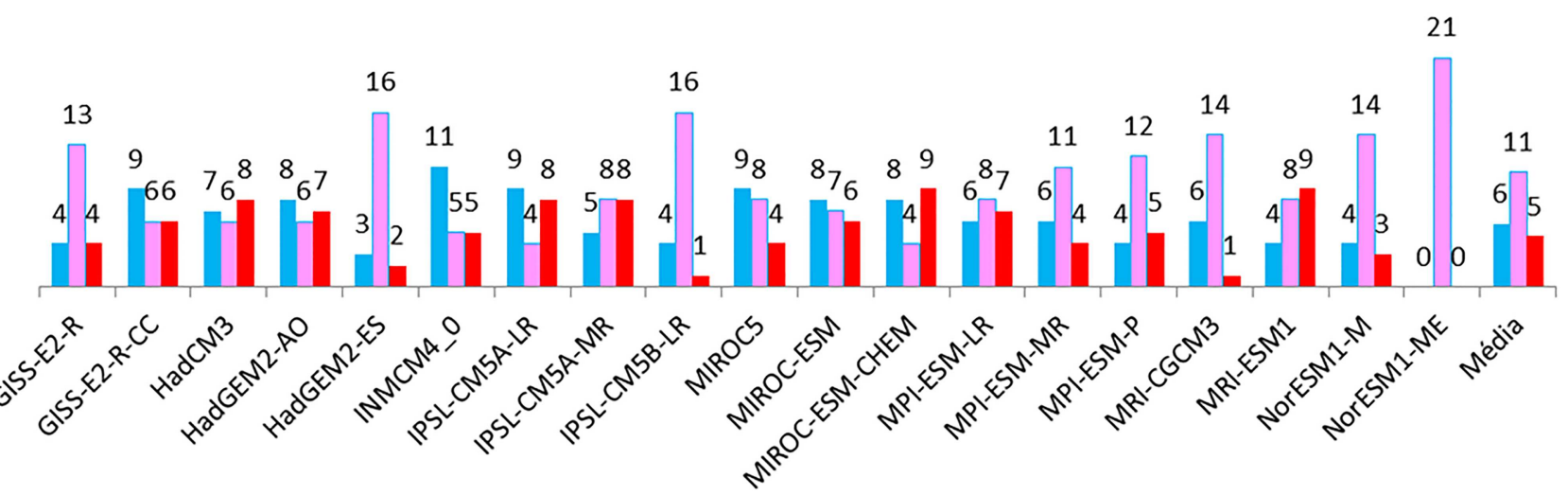

Figura 3. - Eventos do gradiente meridional de anomalias de TSM no Atlântico Tropical para a estação de verão dos dados do ERSST e dos modelos do CMIP5 (1979-1999). As barras vermelhas, azuis e rosas apresentam a quantidade de dipolos negativos, positivos e neutros, respectivamente. a) ERSST e os modelos do CMIP5 b) Restante dos modelos e a média dos mesmos.

De acordo com esta tabela dois dos quarenta e um modelos do CMIP5 apresentaram um desempenho aceitável. Observa-se que o GFDL-ESM2G, com ID igual a 0,99 , teve um desempenho muito bom correlação perfeita. O segundo foi o CSIRO_MK3_6, com ID igual a 0,72 cujo desempenho foi classificado como bom.

\section{Conclusões e Recomendações}

Os resultados mostraram que houve uma melhora no desempenho dos modelos em simular o índice do gradiente meridional de anomalias de TSM no Atlântico Tropical do CMIP5 para os modelos do CMIP3. No verão, para o CMIP3 o modelo com melhor simulação dos valores do gradiente meridional de anomalias de TSM foi o IPSL_CM4 com um ID de 0,67 classificado com índice de bom desepenho. Para o CMIP5 nessa estação de verão, um maior número de modelos apresentou ID com valores classificados de bom a ótimo. Ressaltam-se os modelos CCCMA_CGCM3_1_T63 e o GFDL_CM2_1, com índices de desempenho muito bom, valores de ID superiores a 0,75 .

Entre os modelos do CMIP5 o GFDL-ESM2G foi o que apresentou um maior ID com 0,96 para a estação de outono para o gradiente meridional de anomalias de TSM.

Para estudos futuros sugere-se analisar a sensibilidade desses modelos acoplados do CMIP3 e CMIP5 em simular as características espaciais do gradiente meridional de anomalias de TSM do Atlântico Tropical, e analisar outras variáveis físicas dos modelos (ventos, fluxos de calor latente e sensível e evaporação), variáveis que têm influência direta nas características da TSM. 
Tabela 5 - Estatísticas para a estação de verão, gradiente meridional de anomalias de TSM, entre os modelos do CMIP5 e os dados do ERSST (1979-1999). Os modelos com maiores IDs e coeficiente de correlação estão marcados em amarelo. As linhas em amarelo indicam quando o coeficiente de correlação foi significativo segundo teste t Student a 95\%.

\begin{tabular}{|c|c|c|c|c|}
\hline $\begin{array}{c}\text { Modelos do AR5 - } \\
\text { Verão }\end{array}$ & $\begin{array}{c}\text { Erro } \\
\text { Médio }\left({ }^{\circ} \mathrm{C}\right)\end{array}$ & $\begin{array}{c}\text { Erro } \\
\text { Quadrático }\left({ }^{\circ} \mathrm{C}\right)\end{array}$ & ID & Correlação \\
\hline BCC_CSM1_1 & 0,04 & 0,12 & $-0,88$ & $-0,90$ \\
\hline BCC_CSM1_1m & $-0,08$ & 0,14 & 0,84 & 0,86 \\
\hline BNU_ESM & $\mathbf{0 , 0 1}$ & 0,14 & 0,67 & 0,69 \\
\hline CanCM4 & 0,01 & 0,11 & $-0,21$ & $-0,21$ \\
\hline CanNESM2 & 0,06 & 0,10 & $-0,76$ & $-0,77$ \\
\hline CCSM4 & $-0,01$ & 0,08 & $-0,29$ & $-0,30$ \\
\hline CESM1-BGC & $-0,01$ & $\mathbf{0 , 0 7}$ & $-0,51$ & $-0,52$ \\
\hline CESM1-CAN5,1 & $\mathbf{0 , 0 0}$ & 0,05 & $-0,47$ & $-0,48$ \\
\hline CMCC_CESM & $-0,10$ & 0,11 & 0,87 & $\mathbf{0 , 8 9}$ \\
\hline CMCC_CM & $-0,03$ & 0,09 & 0,23 & 0,23 \\
\hline CMCC_CMS & 0,04 & 0,05 & $-0,47$ & $-0,48$ \\
\hline CMCC_CM5 & $-0,04$ & 0,08 & 0,77 & 0,78 \\
\hline CMCC_CM5,2 & 0,04 & 0,10 & $-0,81$ & $-0,82$ \\
\hline CSIRO_MK3_6 & $-0,02$ & 0,10 & 0,17 & 0,17 \\
\hline FGOALS-g2 & 0,06 & 0,15 & $-0,88$ & $-0,90$ \\
\hline FIO-ESM & $\mathbf{0 , 0 1}$ & 0,10 & $-0,91$ & $-0,92$ \\
\hline EC_EARTH & 0,06 & 0,12 & $-0,68$ & $-0,70$ \\
\hline GFDL-CM2p1 & 0,05 & 0,11 & $-0,82$ & $-0,84$ \\
\hline GFDL-CM3 & 0,07 & 0,15 & 0,08 & 0,08 \\
\hline GFDL-ESM2G & $\mathbf{0 , 0 7}$ & $\mathbf{0 , 0 3}$ & $-0,92$ & $-0,92$ \\
\hline GISS-E2-H & 0,01 & $\mathbf{0 , 0 3}$ & 0,61 & 0,61 \\
\hline GISS-E2-H-CC & $\mathbf{0 , 0 1}$ & $\mathbf{0 , 0 3}$ & $-0,53$ & $-0,53$ \\
\hline GISS-E2-R & $\mathbf{0 , 0 3}$ & 0,05 & $-0,90$ & $-0,91$ \\
\hline GISS-E2-R-CC & 0,10 & $\mathbf{0 , 3 1}$ & $-0,90$ & $-0,94$ \\
\hline HadCM3 & $-0,09$ & 0,23 & 0,63 & 0,66 \\
\hline HadGEM2-AO & $\mathbf{0 , 0 7}$ & 0,19 & $-0,45$ & $-0,46$ \\
\hline HadGEM2-ES & $\mathbf{0 , 0 2}$ & $\mathbf{0 , 0 3}$ & $-0,63$ & $-0,63$ \\
\hline INMCM4_0 & 0,06 & 0,18 & $-0,66$ & $-0,68$ \\
\hline IPSL-CM5A-LR & $-0,01$ & 0,18 & 0,74 & 0,77 \\
\hline IPSL-CM5A-MR & 0,02 & 0,23 & $-0,24$ & $-0,25$ \\
\hline IPSL-CM5B-LR & 0,05 & 0,06 & $-0,35$ & $-0,36$ \\
\hline MIROC5 & 0,08 & 0,19 & $-0,12$ & $-0,12$ \\
\hline MIROC-ESM & $-0,04$ & 0,13 & 0,66 & 0,67 \\
\hline $\begin{array}{l}\text { MIROC-ESM- } \\
\text { CHEM }\end{array}$ & $-0,01$ & 0,12 & $-0,45$ & $-0,46$ \\
\hline MPI-ESM-LR & 0,01 & 0,08 & $-0,02$ & $-0,02$ \\
\hline MPI-ESM-MR & $\mathbf{0 , 0 1}$ & 0,06 & $-0,63$ & $-0,64$ \\
\hline MPI-ESM-P & 0,02 & 0,06 & $-0,20$ & $-0,21$ \\
\hline MRI-CGCM3 & 0,10 & $\mathbf{0 , 0 5}$ & $-0,88$ & $-0,89$ \\
\hline MRI-ESM1 & $-0,07$ & 0,09 & 0,20 & 0,21 \\
\hline NorESM1-M & $\mathbf{0 , 0 1}$ & 0,08 & $-0,47$ & $-0,48$ \\
\hline NorESM1-ME & $\mathbf{0 , 0 3}$ & $\mathbf{0 , 0 1}$ & $-0,40$ & $-0,40$ \\
\hline Média & 0,01 & 0,11 & $-0,24$ & $-0,25$ \\
\hline
\end{tabular}

Tabela 6 - Estatísticas para a estação de outono, gradiente meridional de anomalias de TSM, entre os modelos do CMIP5 e os dados do ERSST (1979-1999). Os modelos com maiores IDs e coeficiente de correlação estão marcados em amarelo. As linhas em amarelo indicam quando o coeficiente de correlação foi significativo segundo teste t Student a 95\%.

Modelos do AR5 Erro Médio Erro Quadrático ID Correla-

\begin{tabular}{|c|c|c|c|c|}
\hline -Ooutono & $\left({ }^{\circ} \mathrm{C}\right)$ & $\left({ }^{\circ} \mathrm{C}\right)$ & & ção \\
\hline BCC_CSM1_1 & 0,06 & 2,55 & 0,10 & 0,11 \\
\hline BCC_CSM1_1m & $-0,08$ & 2,49 & $-0,22$ & $-0,23$ \\
\hline BNU_ESM & $\mathbf{0 , 0 1}$ & 2,64 & $-0,56$ & $-0,57$ \\
\hline CanCM4 & 0,03 & 2,59 & $-0,34$ & $-0,34$ \\
\hline CanNESM2 & 0,03 & 2,31 & 0,12 & 0,13 \\
\hline CCSM4 & $-0,05$ & 2,27 & $-0,26$ & $-0,27$ \\
\hline CESM1-BGC & $-0,05$ & 2,64 & $-0,49$ & $-0,50$ \\
\hline CESM1-CAN5,1 & $-0,07$ & 2,27 & $-0,23$ & $-0,24$ \\
\hline CMCC_CESM & $-0,10$ & 2,32 & $-0,27$ & $-0,27$ \\
\hline CMCC_CM & $-0,04$ & 2,36 & $-0,10$ & $-0,11$ \\
\hline CMCC_CMS & 0,04 & 1,95 & 0,42 & 0,42 \\
\hline CMCC_CM5 & 0,03 & 2,37 & $-0,26$ & $-0,26$ \\
\hline CMCC_CM5,2 & 0,07 & 1,89 & 0,34 & 0,35 \\
\hline CSIRO_MK3_6 & $-0,02$ & 1,66 & 0,72 & 0,73 \\
\hline FGOALS-g2 & 0,05 & 2,15 & 0,34 & 0,35 \\
\hline FIO-ESM & 0,02 & 2,36 & 0,02 & 0,02 \\
\hline EC_EARTH & $\mathbf{0 , 0 2}$ & 2,15 & 0,36 & 0,37 \\
\hline GFDL-CM2p1 & 0,04 & 2,04 & 0,27 & 0,28 \\
\hline GFDL-CM3 & 0,05 & 1,85 & 0,24 & 0,25 \\
\hline GFDL-ESM2G & $\mathbf{0 , 0 2}$ & 2,14 & 0,97 & 0,96 \\
\hline GISS-E2-H & 0,08 & 2,23 & 0,08 & 0,08 \\
\hline GISS-E2-H-CC & $-0,01$ & 2,22 & $-0,29$ & $-0,29$ \\
\hline GISS-E2-R & 0,00 & 2,08 & 0,36 & 0,37 \\
\hline GISS-E2-R-CC & 0,03 & 2,23 & 0,20 & 0,20 \\
\hline HadCM3 & 0,01 & 2,25 & 0,07 & 0,07 \\
\hline HadGEM2-AO & $-0,11$ & 2,66 & 0,18 & 0,19 \\
\hline HadGEM2-ES & 0,05 & 2,30 & $-0,10$ & $-0,10$ \\
\hline INMCM4_0 & 0,01 & 2,09 & 0,35 & 0,36 \\
\hline IPSL-CM5A-LR & $\mathbf{0 , 0 8}$ & 2,13 & 0,41 & 0,42 \\
\hline IPSL-CM5A-MR & $-0,04$ & 1,83 & 0,42 & 0,42 \\
\hline IPSL-CM5B-LR & $\mathbf{0 , 0 1}$ & 2,01 & 0,49 & $\mathbf{0 , 5 0}$ \\
\hline MIROC5 & 0,05 & 2,32 & $-0,23$ & $-0,24$ \\
\hline MIROC-ESM & 0,10 & 2,21 & 0,24 & 0,24 \\
\hline $\begin{array}{l}\text { MIROC-ESM- } \\
\text { CHEM }\end{array}$ & $-0,01$ & 1,81 & 0,13 & 0,13 \\
\hline MPI-ESM-LR & 0,05 & 2,33 & $-0,04$ & $-0,05$ \\
\hline MPI-ESM-MR & 0,01 & 2,23 & $-0,28$ & $-0,29$ \\
\hline MPI-ESM-P & $-0,01$ & 2,38 & $-0,17$ & $-0,17$ \\
\hline MRI-CGCM3 & 0,06 & 2,44 & $-0,06$ & $-0,06$ \\
\hline MRI-ESM1 & 0,10 & 2,20 & 0,15 & 0,15 \\
\hline NorESM1-M & $-0,06$ & 1,86 & 0,49 & $\mathbf{0 , 5 0}$ \\
\hline NorESM1-ME & $-0,01$ & 2,96 & $-0,75$ & $-0,76$ \\
\hline Média & 0,01 & 2,24 & 0,07 & 0,07 \\
\hline
\end{tabular}




\section{a) Dipolo Meridional - Outono do AR5}

घ Quantidade de dipolo positivo $\quad \square$ Quantidade de dipolo neutro $\quad$ Quantidade de dipolo negativo

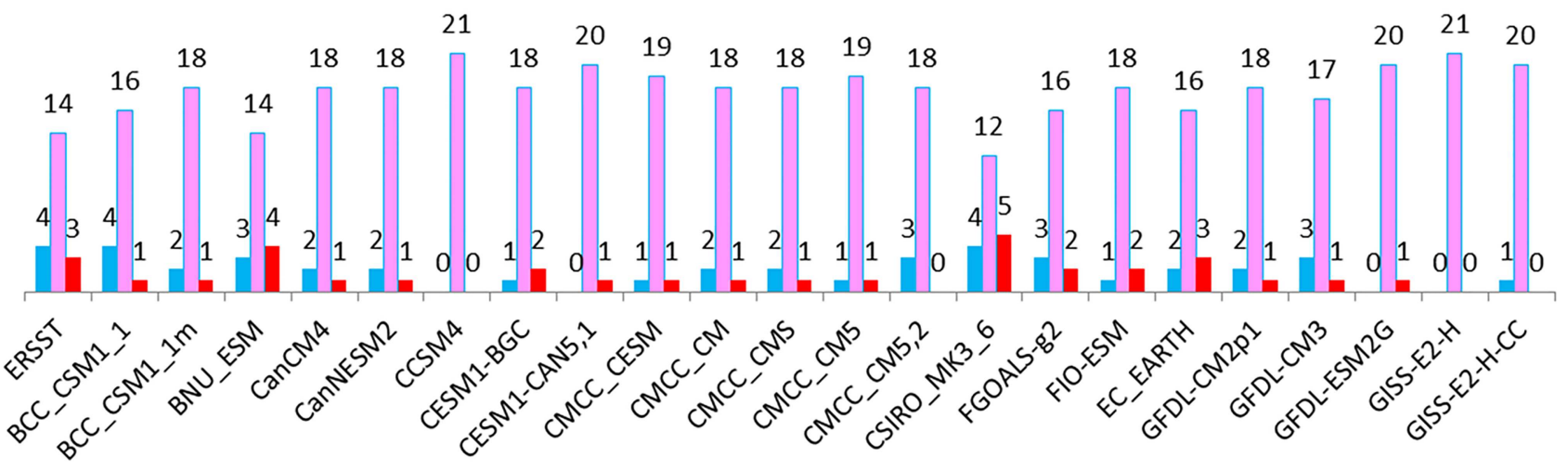

b) Dipolo Meridional - Outono do AR5

Q Quantidade de dipolo positivo $\square$ Quantidade de dipolo neutro $\quad$ Quantidade de dipolo negativo

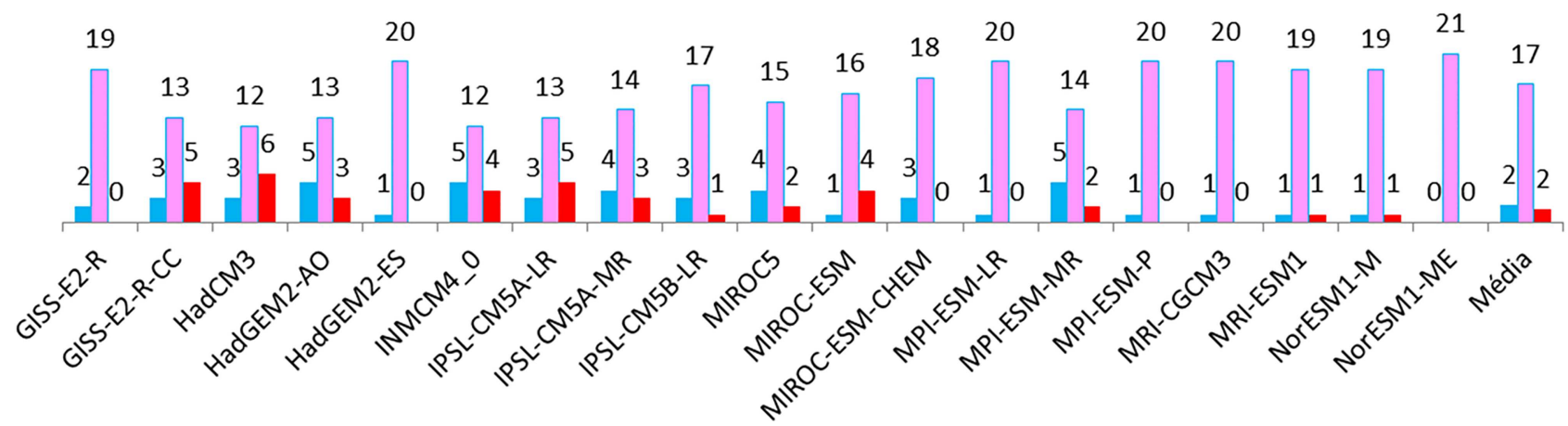

Figura 4. - Eventos do gradiente meridional de anomalias de TSM no Atlântico Tropical para a estação de outono dos dados do ERSST e dos modelos do CMIP5 (1979-1999). As barras vermelhas, azuis e rosas apresentam a quantidade de dipolos negativos, positivos e neutros, respectivamente. a) ERSST e os modelos do CMIP5 b) Restante dos modelos e a média dos mesmos.

\section{Agradecimentos}

As análises desse estudo fazem parte da Dissertação de Mestrado da autora Érika A. de Assis, sob orientação do Prof. Dr. José Maria Brabo Alves apresentada no Mestrado Acadêmico de Ciências Físicas Aplicadas (MACFA) da Universidade Estadual do Ceará (UECE).

\section{Referências}

ALVES, J.M.B.; CAMPOS, J.N.B.; SERVAIN, J. Reservoir Manajament Using Coupled Atmospheric and Hydrological Models: The Brazilian Semi-Arid Case. Water Resource Management, v. 26, n. 5, p.1365-1385. 2012.

ALVES, J.M.B.; CHAVES, R. R.; SERVAIN J.; SOUZA E.B.; SILVA E.M.; SANTOS, A.C.S.; BARBOSA, A.C.B.; OLIVEIRA, C.J.; SOMBRA, S.S.; COSTA, A.A.; PINHEIRO, F.G.M. Modelos acoplados do IPCC-AR4 e o gradiente meridional de temperatura da superfície do mar no Atlântico Tropical: relações com a precipitação no norte do nordeste do Brasil. Revista Brasileira de Meteorologia, v. 29, n. 4, p. 568-578, 2014.

ALVES, J.M.B.; SERVAIN, J.; CAMPOS, J.N. Relationship between ocean climatic variability and rain-fed agriculture in northeast Brazil. Climate Research, v. 38, n. 3, p. 225236, 2009.

ASHOK, K., GUAN, Z.; YAMAGATA, T. Influence of the Indian Ocean dipole on the Australian winter rainfall. Geophysical Research Letters, v. 30, n. 15, p. 1824-1827, 2003.

CAVALCANTI, I.F.A.; FERREIRA, M.; ASSUNçãO, F. da S. D.; GERTRUDES, A.; SILVA, J. Tempo e Clima no Brasil. São Paulo: Oficina de Textos, p. 182. 2009.

CHIANG, J.C. H.; VIMONT, D.J. Analogous Pacific and Atlantic meridional modes of tropical atmosphere-ocean variability, Journal of Climate, v. 17, n. 21, p. 4143-4158, 2004. 
ENFIELD, D.B.; MESTAS-NUñEZ, A.M.; MAYER D.A.; CIDSERRANO L. How ubiquitous sis the dipole relationship in tropical Atlantic sea surface temperatures? Journal of Geophysical Research, v. 104 n. 4, p. 7841-7848, 1999.

GUAN, Z; YAMAGATA, T. The unusual summer of 1994 in East Asia: IOD teleconnections. Geophysical Research Letters, v. 30, n. 15, p. 1544-1547, 2003.

HASTENRATH, S. Interannual variability and annual cycle: mechanisms of circulation and climate in the tropical Atlantic. Monthly Weather Review, v. 112, n. 6, p. -1107, 1984.

HUANG, B.; BANZON, V.F.; P.; FREEMAN, E.; LAWRIMORE, J.; LIU, W.; PETERSON, T.C.; SMITH, T. M.; THORNE, P.W.; WOODRUFF, S.D.; ZHANG, H-M.LIU, W.; LAWRIMORE, J.; BANZON, V.; ZHANG, H-M. Extended Reconstructed Sea Surface Temperature Version 4 (ERSST.v4). Part I: Upgrades and Intercomparisons. Journal of Climate, v. 28, n. 3, p. 911-930, 2015.

IPCC, Climate Change 2013: The Physical Science Basis. Contribution of Working Group I to the Fifth Assessment Report of the Intergovernmental Panel on Climate Change [Stocker, T.F.; D. Qin; G.-K, Plattner, M.; Tignor, S.K; Allen, J.; Boschung, A.; Nauels, Y.; Xia, V.; Bex, P.M. Midgley (eds.)]. Cambridge University Press, Cambridge, 1535 pp., 2013.

IPCC, Summary for Policymakers. Climate Change 2007: The Physical Science Basis. Contribution of Working Group I to the Fourth Assessment Report of the Intergovernmental Panel on Climate Change [Solomon, S.D.; Qin, M.; Manning, Z.; Chen, M.; Marquis, K.B.; Averyt, M.; Tignor, H.L. Miller (eds.)]. Cambridge University Press, Cambridge, 2007.

MEEHL, G.A.; COVEY, C.; TAYLOR, K.E.; DELWORTH, T.; STOUFFER, R.J.; LATIF, M.; McAVANEY, N.; MITCHELL, J.F.B. The WCRP CMIP3 multimodel dataset: a new era in climate change research. Bull. Amer. Meteor. Soc., v. 88. n. 9, p. 1383-1394. 2007.

NNAMCHI, H.C.; LI, J. Influence of the South Atlantic Ocean dipole on West African summer precipitation, Journal of Climate, v. 24, n. 4, p. 1184-1197, 2011.

NOBRE, P.; SHUKLA, J. Variations of sea surface temperatures, wind stress, and rainfall over the tropical over the tropical Atlantic and South America. Journal of Climate, v. 9, n. 10, p. 2464-2479. 1996.
SAJI, N.H.; GOSWAMI, B.N.; VINAYACHANDRAN, P.N.; YAMAGATA, T.A. Dipole mode in the tropical Indian Ocean. Nature, v. 401, p. 360-363, 1999.

SERVAIN, J. Simple climatic indices for the tropical Atlantic Ocean and some applications. Journal of Geophysical Research, v. 96, n. C8, p. 15137-15146, 1991.

SERVAIN, J.; WAINER, I.; McCREARY, J.P.; DESSIER, J.R.A. Relationship between the equatorial and meridional of climate variability in the Tropical Atlantic. Geophysics Res Lett., v. 26, n. 4, p. 485-488, 1999.

SMITH, T.M.; REYNOLDS, R.W. Bias corrections for historic sea surface temperatures based on marine air temperatures. Journal of Climate, v. 15, n. 1, p. 73-87, 2002.

SMITH, T.M.; REYNOLDS, R.W.; PETERSON, T.C.; LAWRIMORE J. Improvements to NOAA's historical merged land-ocean temperature analysis (1880-2006). Journal of Climate, v. 21, n. 10, p. 2283-2296, 2008.

TANIMOTO, Y; XIE, S. - P. Inter-hemispheric decadal variations in SST, wind surface, heat flux and cloud cover over the Atlantic Ocean. Journal of Meteorological of Society of Japan, v. 80, n. 5, p. 1199-1219. 2002.

TASCHETO, A.S.; WAINER. I. The impact of the subtropical South Atlantic SST South American precipitation. Annales Geophysical, v. 26, n. 11, p. 3457-3476. 2008.

TAYLOR, K.E.; STOUFFER, R.J.; MEEHL, G.A. An overview of CMIP5 and the experiment design. Bull Amer Meteor Socie, v. 3, n. 4., p. 465-498. 2012.

TAYLOR, A. M., REBY, D. e MCCOMB, K. Why Do Large Dogs Sound More Aggressive to Human Listeners: Acoustic Bases of Motivational Misattributions. Journal of Ethology, v.116, n. 12, p. 1155-1162, 2010.

WILLMOTT, C.J.; DAVIS, R.E.; FEDDEMA, J.J.; JOHANNES, J.; KLINK, K.M.; LEGATES, D.R.; ROWE, C.M.; ACKLESON, S.G.; O'DONNELL, J. Statistics for the Evaluation and Comparison of Models. Journal of Geophysical Research, v. 90, n. C5, p. 8995-9005, 1985.

WITTE, R. S.; WITTE, J. S. Estatística, $7^{\text {a }}$ edição, LTC, Rio de Janeiro, 2005.

This is an Open Access article distributed under the terms of the Creative Commons Attribution License, which permits unrestricted use, distribution, and reproduction in any medium, provided the original work is properly cited. 